\title{
一维实 $\phi^{3}+\phi^{4}$ 场在有限温度下围绕 孤子的量子起伏
}

\author{
陈 苏 卿 \\ (复旦大学物理系,上海)
}

对有孤子的一维场论及其在有限温度下的行为的研究,近年来吸引了不少人的注意. Su、 Schrieffer 和 Heeger ${ }^{[1]}$ 研究一维聚乙炔的导电机制, 指出顺式聚乙炔的 $A$ 相的能量略高于 $B$ 相,基态没有简并。

前二年,在 $\mathrm{Su} 、 \mathrm{Bi} 、 \mathrm{Ni}^{[2]}$ 的工作中采用动量守恒的实时格林函数方法讨论了有简并基态的 $\phi^{4}$ 场. 接着,在 Chen 和 $\mathrm{Ni}^{[3]}$ 的工作中，引入了动量不守恒的实时格林函数并用它来讨论了 一些场. 有趣的是, 引入动量不守恒的格林函数会导致孤子的零模运动的自然出现. 本工作 将把这个方法用来讨论 $\phi^{3}+\phi^{4}$ 场，它相当于顺式聚乙炔的情形.

$\phi^{3}+\phi^{4}$ 场的拉氏密度为

$$
\mathscr{L}=\frac{1}{2}\left(\frac{\partial \phi}{\partial t}\right)^{2}-\frac{1}{2}\left(\frac{\partial \phi}{\partial x}\right)^{2}+\frac{m_{b}^{2}}{4} \phi^{2}-\frac{g_{b}^{2}}{4} \phi^{4}-\frac{\lambda_{b}}{3} \phi^{3},
$$

令 $\phi(x, t)=\phi_{s}\left(\frac{x-v t}{\sqrt{1-v^{2}}}\right), x^{\prime}=\frac{x-v t}{\sqrt{1-v^{2}}}$, 则经典场方程为

$$
\frac{d^{2} \phi_{s}}{d x^{2}}+\frac{m_{b}^{2}}{2} \phi_{s}-g_{b}^{2} \phi_{s}^{3}-\lambda_{b} \phi_{s}^{2}=0,
$$

我们会看到,这个方程的解存在, 称为孤子解. 为了讨论场在孤子背景上的量子起伏, 我们首 先将 $\phi$ 场量子化:

$$
\hat{\phi}(x, t)=\Sigma_{k} \frac{1}{\sqrt{2 L \omega_{k}}}\left(a_{k}(t)+a_{-k}^{+}(t)\right) e^{i k x},
$$

其中 $\omega_{k}=\sqrt{k^{2}+\mu^{2}}$, 质量 $\boldsymbol{\mu}$ 是任意的. 用 $\operatorname{Coleman}^{[4]}$ 的办法重正化, 令 $\hat{\phi}=\hat{\phi}^{\prime}-a$, 则体系 的哈密顿量为

$$
H=\int N_{\mu}\left[\frac{1}{2} \hat{x}^{\prime 2}+\frac{1}{2}\left(\frac{\partial \hat{\phi}^{\prime}}{\partial x}\right)^{2}+\frac{1}{4} g^{2} \hat{\phi}^{\prime 4}+\frac{\lambda}{3} \hat{\phi}^{\prime 3}-\frac{m^{2}}{4} \hat{\phi}^{\prime 2}+D_{0}\right] d x,
$$

其中 $\boldsymbol{a}$ 由要求 $\hat{\phi}^{\prime}$ 的系数为零而定, 满足

$$
-g_{b}^{2} a^{3}+\lambda_{b} a^{2}+\left(\frac{1}{2} m_{b}^{2}-\frac{3 g_{b}^{2}}{2 \pi} \ln \frac{2 \Lambda}{\mu}\right) a=0,
$$

可以估计 $a, \lambda_{b}, m_{b}$ 的数量级为

$$
a \sim\left(\ln \frac{2 \Lambda}{\mu}\right)^{1 / 2}, \lambda_{b} \sim g^{2}\left(\ln \frac{2 \Lambda}{\mu}\right)^{1 / 2}, m_{b} \sim g\left(\ln \frac{2 \Lambda}{\mu}\right),
$$

本文 1985 年 3 月 16 日收到. 
下面让我们在孤子静此的坐标系内把 $\hat{\phi}^{\prime}$ 分解为

$$
\begin{gathered}
\hat{\phi}^{\prime}=\phi_{s}+\hat{\phi}, \\
\hat{\psi}=\sum_{k} \frac{1}{\sqrt{2 L \omega_{k}}}\left(C_{k}+C_{-k}^{+}\right) e^{i k x},
\end{gathered}
$$

这里的 $\phi_{S}$ 已不含时间了. 把上式代人 (4)式并引人配对近似 ${ }^{[2,3]}$, 则哈密顿量约化为

$$
\begin{aligned}
& H=H_{0}+H_{1}+H_{2}+H_{2}^{\prime}+H_{2}^{\prime \prime}, \\
& H_{0}=\int d x\left[\frac{1}{2}\left(\frac{\partial \phi_{S}}{\partial x}\right)^{2}+\frac{g^{2}}{4} \phi_{S}^{4}+\frac{\lambda}{3} \phi_{S}^{3}-\frac{m^{2}}{4} \phi_{S}^{2}\right], \\
& H_{1}=\int d x\left[-\frac{\partial^{2} \phi_{S}}{\partial x^{2}}+g^{2} \phi_{S}^{3}+\lambda \phi_{S}^{2}-\frac{M^{2}}{2} \phi_{S}\right] \hat{\psi}, \\
& H_{2}=\Sigma_{k}\left(\frac{1}{4} \frac{k^{2}}{\omega_{k}}-\frac{\omega_{k}}{4}-\frac{M^{2}}{8 \omega_{k}}\right)\left(C_{k}^{+} C_{k}^{+}+C_{k} C_{\ddot{k}}\right)+\Sigma_{k}\left(\frac{k^{2}}{2 \omega_{k}}+\frac{\omega_{k}}{2}-\frac{M^{2}}{4 \omega_{k}}\right) C_{k}^{+} C_{k}, \\
& H_{2}^{\prime}=\Sigma_{k_{1} k_{2}} \frac{3}{2} g^{2} \int d x \cdot \phi_{S}^{2}(x) \frac{1}{L \sqrt{4 \omega_{1} \omega_{2}}} e^{i\left(k_{1}+k_{2}\right) x}\left(C_{k_{1}}^{+} C_{k_{2}}^{+}+C_{k_{1}}^{+} C_{k_{2}}+C_{k_{2}}^{+} C_{k_{1}}+C_{k_{1}} C_{k_{2}}\right), \\
& H_{2}^{\prime \prime}=\Sigma_{k_{1} k_{2}} \lambda \int d x \cdot \phi_{S} \frac{1}{L \sqrt{4 \omega_{1} \omega_{2}}}=e^{i\left(k_{1}+k_{2}\right) x}\left(C_{\dot{k}_{1}}^{+} C_{k_{2}}^{+}+C_{k_{1}}^{+} C_{k_{2}}+C_{k_{2}}^{+} C_{k_{1}}+C_{k_{1}} C_{k_{2}}\right), \\
& M^{2}=m^{2}-12 g v, \\
& \nu=\Sigma_{k} \frac{1}{2 L \omega_{k}}\left(\left\langle C_{k} C_{\bar{k}}+C_{k}^{+} C_{k}\right\rangle\right),
\end{aligned}
$$

这里 $\bar{k}=-k$. 把含 $\phi_{s}$ 的 $H_{2}^{\prime}, H_{2}^{\prime \prime}$ 进一步配对, 与 $H_{0}$ 合并, 得

$$
H_{0}^{\prime}=\int d x\left[\frac{1}{2}\left(\frac{\partial \phi_{s}}{\partial x}\right)^{2}+\frac{g^{2}}{4} \phi_{S}^{4}+\frac{\lambda}{3} \phi_{S}^{3}-M^{2} \phi_{S}^{2}+2 \lambda \nu \phi_{S}\right],
$$

由 $\frac{\delta H_{0}^{\prime}}{\delta\left(\phi_{S}(y)\right)}=0$, 我们得到 $\phi_{S}$ 满足的方程

$$
\frac{\partial^{2} \phi_{s}}{\partial x^{2}}+\frac{M^{2}}{2} \phi_{s}-g^{2} \phi_{s}^{3}-\lambda \phi_{s}^{2}-2 \lambda \nu=0,
$$

当量子起伏为零即零温时, $v$ 为零. 注意到在孤子静止系中的 $x$ 与 (2) 式中的 $x^{\prime}$ 一致, 那末 (14)式与(2)式是一致的.

将(14)式积分,并令 $K=\frac{1}{2} \frac{g^{2}}{m^{2}}, \tau=-\frac{2}{3} \frac{\lambda}{m^{2}}, \xi=m x$ 得

$$
\left(\frac{d \phi_{S}}{d \xi}\right)^{2}+\left(\frac{1}{2}-12 K \nu\right) \phi_{S}^{2}(\xi)-K \phi_{S}^{4}(\xi)+\tau \phi_{S}^{3}(\xi)-6 \nu \tau \phi_{S}=E,
$$

可以把(15)式看为描写一个粒子运动的力学方程 $T+V=E$,

$$
V\left(\phi_{S}\right)=\left(\frac{1}{2}-12 K \nu\right) \phi_{S}^{2}-K \phi_{S}^{4}+\tau \phi_{S}^{3}-6 \nu \tau \phi_{S},
$$

粒子在势 $V\left(\phi_{S}\right)$ 中运动. 图 1 给出 $V\left(\phi_{S}\right)$ 随 $v$ 的变化.

令 $a$ 是 $V\left(\phi_{S}\right)$ 的各极点处的 $\phi_{S}$ 值，由图可以预料，当粒子能量取图中 $\phi_{S}=d_{1}$ 处的 $V\left(d_{1}\right)$ 值, $E=V\left(d_{1}\right)$, 那末粒子可以在图中用阴影标出的谷中运动, 从而可以予期存在一个 孤子解. 当温度升高, $v$ 增大，谷逐渐变浅直至消失，孤子解就不再存在. 让我们把势的原点 

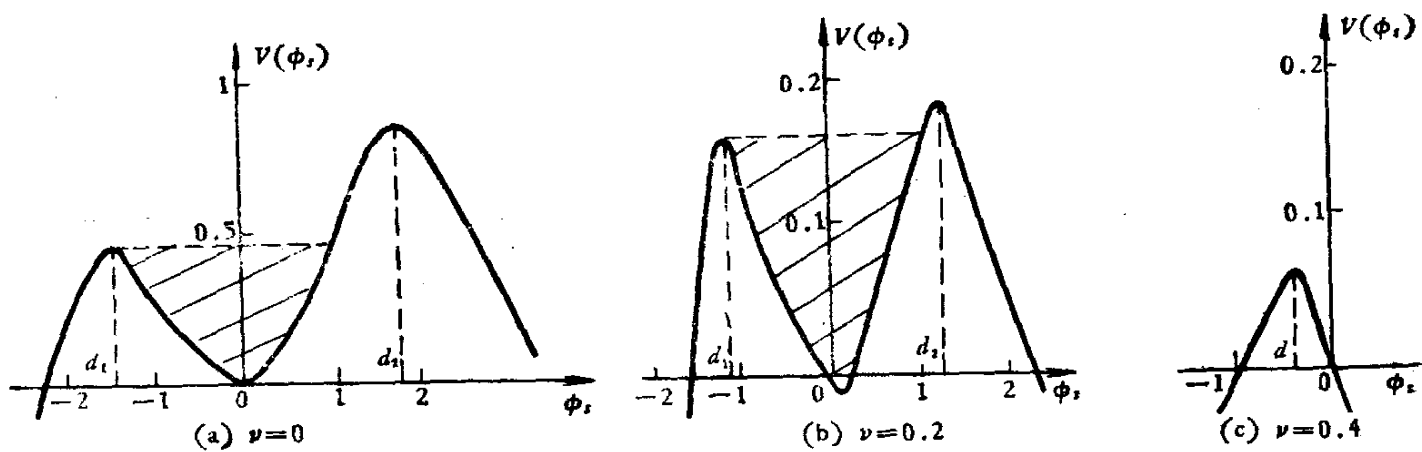

图 $1 V\left(\phi_{S}\right)$ 随 $\nu$ 的变化 $(K=0.1, \tau=0.05, \nu=0,0.2,0.4)$

(图中 $d$ 为 $d_{1}$ )

作一个平移, 移到 $V(d)$ 处, 即令 $\phi_{S}=\phi_{0}+d, E=V(d)$, 那末(15)式化为

$$
T+V\left(\phi_{0}\right)=\left(\frac{d \phi_{0}}{d \xi}\right)^{2}-B \phi_{0}^{4}+D \phi_{0}^{3}-A \phi_{0}^{2}=0,
$$

式中 $B=K, D=\tau-4 K d, A=6 K d^{2}-3 \tau d+12 K \nu-\frac{1}{2} \cdot V\left(\phi_{0}\right)$ 的示意图如图 2 .

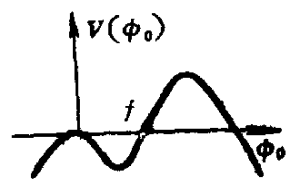

(a) $D>0, A>0$

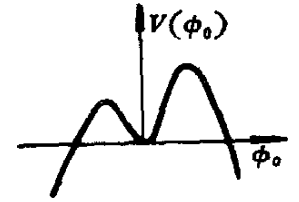

(b) $D>0, A<0$



(c) $D<0, A>0$

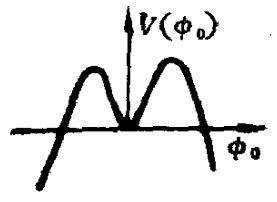

(d) $D<0, A<0$

图 $2 V\left(\phi_{0}\right)$ 在 $D<0$ 和 $D>0$ 时的示意图

由图 2 可知, 当 $A<0$, 在谷内运动的粒子能量将大于零, 从而不产生符合(17)式的解, 这是 与我们不准确地取了 $E=V\left(d_{2}\right)$ 相关的. $V\left(\phi_{0}\right)$ 与横轴的交点 $f$ 为 $0, \frac{D \pm \sqrt{D^{2}-4 A B}}{2 B}$, 于是方程 (17) 式的孤子解可以参照孙、李 ${ }^{[3]}$ 的工作给出为

$$
\phi_{0}(\eta)=\frac{2\left(\frac{1}{f}-\frac{D}{2 A}\right) e^{ \pm \eta}}{\left[\frac{D}{2 A}-\left(\frac{1}{f}-\frac{D}{2 A}\right) e^{ \pm \eta}\right]-\frac{B}{A}},
$$

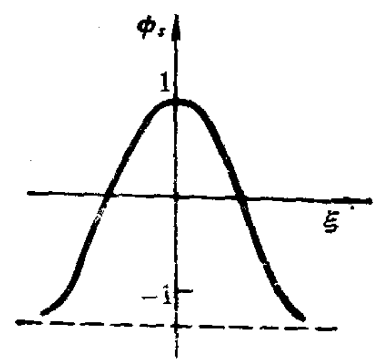

(a) $x=-0.05$

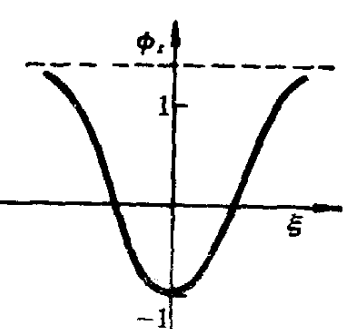

(b) $\tau=0.05$

图 $3 \nu=0, K=0.1, \nu= \pm 0.05$ 时的 $\phi_{S}$ 图 
式中 $\eta=\sqrt{A} \xi$, 士号与 $\eta \geq 0$ 对应. 下面给出 $v=0$ 时 $\phi_{S}\left(=\phi_{0}+d\right)$ 的图(图 3).

为了讨论量子起伏求出元激发谱, 按照文献 [3]引人动量不守恒的格林函数和关联函数为

$$
\begin{gathered}
G_{1}(p, q) \equiv\left\langle\left\langle C_{p} \mid C_{q}^{+}\right\rangle, G_{2}(p, q) \equiv\left\langle\left\langle C^{+}{ }_{p} C_{q}^{+}\right\rangle,\right.\right. \\
\left\langle C_{q}^{+} C_{p}+C_{q}^{+} C_{\bar{p}}^{+}\right\rangle=\frac{(2 \pi)^{2}}{L}-\left(\frac{\omega_{p}}{\omega_{q}}\right)^{\frac{1}{2}} \Sigma_{E_{n}>0}\left[\frac{\frac{1}{2}\left(1+\omega_{q}\right) / E_{n}}{e^{\beta I_{n}}-1} \frac{\frac{1}{2}\left(1-\omega_{q}\right) / E_{n}}{e^{-\beta E_{n}}-1}\right] \tilde{u}_{n}(p) \tilde{u}_{n}^{*}(q),
\end{gathered}
$$

式中 $\tilde{u}_{n}(p)$ 的付里叶变换 $u_{n}(x)$ 是满足下面本征值方程的解

$$
\begin{gathered}
\left(E_{n}^{2}-\hat{H}(x)\right) u_{n}(x)=0, \\
\hat{H}(x)=-\frac{d^{2}}{d x^{2}}+3 g^{2} \phi_{S}^{2}+2 \lambda \phi_{S}-\frac{M^{2}}{2},
\end{gathered}
$$

把 $E_{n}=0$ 和 $u_{0}(x)=\frac{d \phi_{s}}{d x}$ 代入(21) 式,并积分之得

$$
\phi_{s}^{\prime \prime}+\frac{M^{2}}{2} \phi_{s}-\lambda \phi_{s}^{2}-g^{2} \phi_{s}^{3}=\text { const }
$$

显然 (23) 式与 $\phi_{S}$ 的方程 (14)式相一致, 从而证明了在 $\phi^{3}+\phi^{4}$ 模型中 $E_{n}=0$ 的零模解 $\frac{d \phi_{S}}{d x}$ 再次自然地出现。

由于除零模解以外, 找方程 (22) 的本征解的解析解存在困难，(20)式中的 $E_{n}$ 用 (19)式的 $p=q$ 的动量守恒格林函数 ${ }^{[2]}$ 的谱表示来得到. 在 $\phi^{3}+\phi^{4}$ 模型, 我们有

$$
\begin{gathered}
\Omega_{p}=\frac{p^{2}}{2 \omega_{p}}+\frac{\omega_{p}}{2}-\frac{M^{2}}{4 \omega_{p}}+\frac{3 g^{2}}{2 L \omega_{p}} \int d x \phi_{S}^{2}(x)+\frac{\lambda}{L\left(\omega_{p} p\right.} \int d x \cdot \phi_{S}(x), \\
\Delta_{p}=\Omega_{p}-\omega_{p} .
\end{gathered}
$$

当积分区间 $L \rightarrow \infty$ 时,我们算得元激发能量 $E_{n}(p)\left(\equiv E_{p}\right)$ 为

$$
E_{p}^{2}=\Omega_{p}^{2}-\Delta_{p}^{2}=p^{2}+M^{2}+\frac{\lambda^{2}}{2 g^{2}} \mp \frac{\lambda \sqrt{\lambda^{2}+2 g^{2} M^{2}}}{2 g^{2}},
$$

其中干号分别对应 $\tau \lessgtr 0$. 现在让我们取重整化常数 $\mu$ 为

$$
\mu^{2}=M^{2}+\frac{\lambda^{2}}{2 g^{2}} \mp \frac{\lambda \sqrt{\lambda^{2}+2 g^{2} M^{2}}}{2 g^{4}},
$$

使得 $\omega_{p}=\sqrt{p^{2}+\mu^{2}}=E_{p}$, 同时把 (20)式中的 $\tilde{u}_{n}(p)$ 近似地取为平面波 $\frac{1}{\sqrt{L}} e^{i p_{n} x}$ 的傅里 叶变换, 那末

$$
\begin{aligned}
\nu & =\frac{1}{2 \pi} \int_{0}^{\infty} \frac{d p}{\omega_{p}}\left\langle C_{p}^{+} C_{p}+C_{p}^{+} C_{\bar{p}}^{+}\right\rangle \\
& =\frac{1}{2 \pi}\left[\frac{\pi T}{2 \mu}+\frac{1}{2} \ln \frac{\mu}{4 \pi T}+\frac{1}{2} \gamma+o\left(\frac{\mu^{2}}{T^{2}}\right)\right],
\end{aligned}
$$

其中 $\gamma=0.5772$ 是欧勒常数. 把(28)式代入(11)式,得到

$$
M^{2}=m^{2}-3 g^{2} T / \mu-3 g^{2} / \pi\left[\ln \frac{\mu}{4 \pi T}+\gamma\right],
$$

由(27)式可知当 $M=M(T)$, 则 $\mu=\mu(T)$, 相应地 $a, \lambda$, 和 $m$ 也与 $T$ 有关. 由(5)式和(6)式 
知, $a$ 和 $\lambda$ 随温度的变化可略. 而

$$
m^{2}=m_{0}^{2}+\frac{3 g^{2}}{\pi} \ln \frac{\mu}{\mu_{0}},
$$

现在 $M(T)$ 可以表示为零温时的参量 $m_{0}, \mu_{0}, g, \lambda$ 和温度 $T$ 的函数. 把(30)式代人(29)式， 并假定 $\lambda \ll g^{2} \ll m_{0}^{2}$, 可以近似地取 $\mu \approx M \mp \frac{\lambda}{2 \sqrt{2} g}$, 其中 (干)号分别对应 $\tau \lessgtr 0$, 于是有

$$
\begin{gathered}
M^{3} \mp \frac{\lambda}{2 \sqrt{2} g} M^{2}-\left[m_{0}^{2}-\frac{3 g^{2}}{\pi}\left(\ln \frac{\mu_{0}}{4 \pi T}+\gamma\right)\right] M+\left\{3 g^{2} T \pm \frac{\lambda}{2 \sqrt{2} g}\right. \\
\left.\left[m_{0}^{2}-\frac{3 g^{2}}{\pi}\left(\ln \frac{\mu_{0}}{4 \pi T}+\gamma\right)\right]\right\}=0,
\end{gathered}
$$

由上式存在二个实根, 其中之一为正根的条件, 导致临界温度 $T_{c}$ 为

$$
\begin{aligned}
T_{c} \cong & \frac{2 m_{0}^{3}}{9 \sqrt{3} g^{2}}\left\{1-\frac{9 g^{2}}{2 \pi m_{0}^{2}}\left(\ln \frac{9 \sqrt{3}}{8 \pi m_{0}^{3}} \mu_{0} g^{2}\right.\right. \\
& \left.\left(\ln \frac{9 \sqrt{3} \mu_{0} g^{2}}{8 \pi m_{0}^{3}}+r\right)\right\} \mp \frac{\lambda m_{0}^{2}}{9 \sqrt{2} g^{3}}\left\{1-\frac{3 g^{2}}{m_{0}^{2} \pi}\right. \\
&
\end{aligned}
$$

式中干号分别对应 $\tau \lessgtr 0$.

我们看到当 $\lambda$ 不为零时, $T_{c}$ 低于 $\phi^{4}$ 的临界温度 ${ }^{[2]}$, 当 $\lambda$ 为零时, 上式与 $\phi^{4}$ 的 $T_{c}$ 表示式一 致.

致谢：作者感谢苏汝链、顾向光同志向作者提供了他们的有关的工作情况.

\section{参考文献}

[1] Su, W. P., Schrieffer, J. R., Heeger, A. F., Phys. Rev., B22(1980), 2099.

[2] Su, R. K., Bi, P. Z., and Ni, G. J., J. Phys. A: Math. Gen., 16(1983), 2445.

[ 3 ] Chen, S. Q, and Ni, G. J., J. Phys. A: Math. Gen., 16(1983), 3493.

[ 4 ] Coleman, S., Phys, Rev., D11(1975), 2088.

[5] 孙金、李洪芳, 科学通报, 24(1979), 10:443。 